\title{
Sum Rate Maximization in the Uplink of Multi-Cell OFDMA Networks
}

\author{
Hina Tabassum*, Zaher Dawy**, and Mohamed Slim Alouini* \\ *Physical Sciences and Engineering Division, King Abdullah University of Science and Technology (KAUST), Thuwal, Saudi Arabia \\ ** Department of Electrical and Computer Engineering, American University of Beirut (AUB), Beirut, Lebanon \\ Email: hina.tabassum@kaust.edu.sa,zd03@aub.edu.lb, slim.alouini@kaust.edu.sa
}

\begin{abstract}
Resource allocation in orthogonal frequency division multiple access (OFDMA) networks plays an imperative role to guarantee the system performance. However, most of the known resource allocation schemes are focused on maximizing the local throughput of each cell, while ignoring the significant effect of inter-cell interference. This paper investigates the problem of resource allocation (i.e., subcarriers and powers) in the uplink of a multi-cell OFDMA network. The problem has a non-convex combinatorial structure and is known to be NP hard. Firstly, we investigate the upper and lower bounds to the average network throughput due to the inherent complexity of implementing the optimal solution. Later, a centralized sub-optimal resource allocation scheme is developed. We further develop less complex centralized and distributed schemes that are well-suited for practical scenarios. The computational complexity of all schemes has been analyzed and the performance is compared through numerical simulations. Simulation results demonstrate that the distributed scheme achieves comparable performance to the centralized resource allocation scheme in various scenarios.
\end{abstract}

Keywords: OFDMA, multi-cell, power control, resource allocation

\section{INTRODUCTION}

In this paper, the problem of scheduling (i.e., determining the active users for a given time interval) and power allocation (i.e., assigning powers to these active users) in a multicell OFDMA network is considered. Solving this problem in an optimal way is cumbersome due to the complexity issues involved in the joint optimization of a large number of continuous and discrete variables, non-convexity of the problem even for the continuous power variables, discrete nature of subcarrier allocations and the interdependency of power and subcarrier allocations.

The sum rate maximization problem is extensively studied for downlink OFDMA networks. However, for the uplink scenario, the problem becomes more challenging due to the individual power constraint at each user. Simply allocating a subcarrier to the user with best channel quality may affect the network performance considerably, as some active users may have better channel gains but low transmission powers on a specific subcarrier. Thus, in order to accomplish near optimal throughput in the uplink, a subcarrier shall be assigned to the user who possess the maximum power-channel gain product [1]-[3]. In [1], a near optimal greedy scheme is proposed which is based on maximizing the marginal rate with an

978-1-4244-9538-2/11/\$26.00(C)2011 IEEE iterative water-filling on the subcarriers. The authors in [2] generalized the strategy presented in [1] by considering utility maximization and developed a polynomial time algorithm to compute an upper bound of the optimal solution. They also verified the near optimality of the solution in [1]. In [3], sum rate maximization is studied with and without proportional fairness and a transmission power-SNR product (PSP) based ranking scheme is developed. The scheme is based on the statistical knowledge of the number of subcarriers assigned to each user. In [4], [5], the authors developed a sub-gradient based scheduling framework to compute the optimal solution of the relaxed problem. In all of the above resource allocation schemes, inter-cell interference (ICI) is not considered. This ignorance may lead to worst performance in the multi-cell scenarios.

Most of the recent work in the context of multi-cell OFDMA networks [6], [7], aims at minimizing the overall transmitted power i.e., linear objective with pre-defined rate constraints. In [8], the authors investigated scaling laws for upper and lower bound of the capacity in asymptotic regime. The results are presented for small downlink networks. Furthermore, in some recent literature, low complexity distributed game theoretic solutions are also studied. However, the schemes are iterative and optimality is not guaranteed [9]. An auction based approach is discussed in [10], where the authors proposed a joint auction and dual decomposition based technique for the resource allocation problem. The technique is asymptotically optimal as the number of subcarriers in every cell goes to infinity. However, this may not be true for finite number of carriers. In summary, all these approaches are suboptimal and no criterion is mentioned to calibrate their performance gap with respect to the optimal solution.

Motivated by the above discussed facts, in this paper, we aim at maximizing the network's throughput without considering any rate constraints. Due to the inherent complexity associated with implementing the optimal solution, an upper (UB) and lower bound (LB) to the network throughput is investigated. The solution in both cases can be considered as distributed if the knowledge of local channel and interfering gains is available. A centralized suboptimal resource allocation scheme is then developed which uses the optimal geometric programming (GP) based power control phase in conjunction with heuristic subcarrier allocation phase. Furthermore, less complex centralized and semi-distributed/distributed schemes 
are proposed. The performance of all the proposed schemes is calibrated using the derived bounds.

The rest of the paper is organized as follows: In Section II, the system model is defined and problem is formulated. In Section III, the bounds are derived and their complexity is analyzed. In Section IV and V, the centralized and semidistributed/distributed resource allocation schemes are presented. Section VI demonstrates numerical results followed by concluding remarks in Section VII.

Notation: Throughout the paper, we denote the sets of real and complex vectors of $N$ elements by $\mathbb{R}^{N}$ and $\mathbb{C}^{N}$, respectively. Matrices are represented using boldface upper case letters while bold face lower case letters are used for vectors.

\section{System Model and Problem Formulation}

A network of $L$ cells with a set of $K_{l}$ users in each cell $l$ is considered. Full reuse of the spectrum is assumed in all the cells, i.e., the reuse ratio is unity. Each BS is assumed to have $N$ orthogonal subcarriers, and each subcarrier can be allocated to a single user per cell. The average throughput per cell is a function of both subcarrier allocation and power allocation variables. The sum rate maximization problem is formulated using the standard Shannon capacity formula $\mathrm{C}_{n, k_{l}, l}=\log _{2}\left(1+\gamma_{n, k_{l}, l}\right)$ where $\mathrm{C}_{n, k_{l}, l}, \gamma_{n, k_{l}, l}$ represents the throughput and signal to interference plus noise ratio (SINR) of $k_{l}^{\text {th }}$ user at $n^{\text {th }}$ subcarrier in cell $l$, respectively:

$$
\begin{aligned}
& \underset{\mathbf{P}_{l}, \mathbf{A}_{l}}{\operatorname{maximize}} \sum_{l=1}^{L} \sum_{k_{l}=1}^{K_{l}} \sum_{n=1}^{N} \alpha_{n, k_{l}, l} \log _{2}\left(1+\frac{p_{n, k_{l}, l} h_{n, k_{l}, l}}{\sigma^{2}+I_{n, l}}\right) \\
& \text { subject to } \sum_{n=1}^{N} \alpha_{n, k_{l}, l}, p_{n, k_{l}, l} \leq \mathrm{P}_{k_{l}, \max }, \forall k_{l}, \forall l \\
& \sum_{k_{l}=1}^{K_{l}} \alpha_{n, k_{l}, l}=1, \forall n, \forall l \\
& \alpha_{n, k_{l}, l} \in[0,1], \forall n, \forall l, \forall k_{l}
\end{aligned}
$$

In (1), $I_{n, l}=\sum_{j=1, j \neq l}^{L} \sum_{k_{j}=1}^{K_{j}} \alpha_{n, k_{j}, j} p_{n, k_{j}, j} g_{n, k_{j}, j l}$ which represents the cumulative interference at $n^{\text {th }}$ subcarrier in cell $l$ from the users in all other cells, $p_{n, k_{l}, l}$ denotes the power transmitted by $k_{l}^{\text {th }}$ user at the $n^{\text {th }}$ subcarrier in cell $l, \alpha_{n, k_{l}, l}$ represents the allocation of $k_{l}^{\text {th }}$ user at the $n^{\text {th }}$ subcarrier in cell $l$ and $h_{n, k_{l}, l}$ is the channel gain of $k_{l}^{\text {th }}$ user at the $n^{\text {th }}$ subcarrier in cell $l$. Constraint (2) implies that the power spent by $k_{l}^{\text {th }}$ user on its allocated subcarriers cannot exceed the maximum available power $\left(P_{k_{l, \max }}\right)$. For each cell, we collect the power allocation variables $p_{n, k_{l}, l}$ in a vector $\mathbf{p}_{n, l}=\left[p_{n, 1}, p_{n, 2}, \ldots ., p_{n, K_{l}}\right]$ and then stack all the vectors in a power matrix $\mathbf{P}_{l}$ of cell $l$ where $\mathbf{P}_{l} \in \mathbb{R}^{N \times K_{l}}$. Constraint (3) restricts the allocation of a subcarrier to only one user. The channel gains $h_{n, k_{l}, l}$ and binary allocation variables $\alpha_{n, k_{l}, l}$ are stacked up similarly in the matrices $\mathbf{H}_{l}$ and $\mathbf{A}_{l}$, respectively, where $\mathbf{A}_{l}, \mathbf{H}_{l} \in \mathbb{R}^{N \times K_{l}}$. Moreover, we define $g_{n, k_{l}, l j}$ as the interfering gain from the $k_{l}^{\text {th }}$ user in cell $l$ to cell $j, \forall j \neq l$ at $n^{\text {th }}$ subcarrier. We collect these interfering gains into a vector $\mathbf{g}_{n, l j}=\left[g_{n, 1, l j}, g_{n, 2, l j} \ldots, g_{n, K_{l}, l j}\right]$ and then stack all the vectors in a matrix $\mathbf{G}_{l j} \in \mathbb{R}^{N \times K_{l}}$.

\section{Bounds ON THE Network Throughput}

\section{A. Lower Bound $(L B)$ on the Optimal Solution}

The LB for the optimum multi-cell throughput can be achieved when the ICI becomes maximum. Observing the dependency of ICI on the subcarrier allocation and power allocation variables, we assume that each user in each cell is transmitting on each subcarrier with its maximum power. The simple LB for the average network throughput taking the worst case ICI into account can be written as follows:

$$
\mathrm{C}\left(\mathbf{A}_{l}, \mathbf{P}_{l}\right) \geq \frac{1}{L} \sum_{l=1}^{L} \sum_{k_{l}=1}^{K_{l}} \sum_{n=1}^{N} \alpha_{n, k_{l}, l} \log _{2}\left(1+\frac{p_{n, k_{l}, l} h_{n, k_{l}, l}}{\sigma^{2}+\xi_{n, l}}\right)
$$

where $\xi_{n, l}=\sum_{j=1, j \neq l}^{L} \sum_{k_{j}=1}^{K_{j}} P_{k_{j}, \max } g_{n, k_{j}, j l}$. However, a more tighter LB can be achieved by using Algorithm 1 where each subcarrier is allocated to the user based on the following criteria:

$$
\alpha_{n, l}=\underset{\mathbf{P}_{l}}{\operatorname{maximize}} Q_{n, k_{l}, l}
$$

where $Q_{n, k_{l}, l}=\frac{p_{n, k_{l}, l} h_{n, k_{l}, l}}{\xi_{n, l}+\sigma^{2}}$ is a factor that takes into account the power-worst SINR product of each user at each subcarrier. Clearly, the LB subcarrier allocations $\mathbf{A}_{l}$ and power allocations $\mathbf{P}_{l}$ in any cell $l$ can then be computed in a distributed way if the knowledge of local channel and interfering gains is available. These allocations are then utilized to determine the LB network throughput using (1). Note that equal power allocation has insignificant performance loss compared to the optimal water-filling [1], [3] thus power equalization is implemented in Algorithm 1.

\section{B. Upper Bound (UB) on the Optimal Solution}

Establishing an upper bound is significantly important in order to calibrate the performance of sub-optimal resource allocation schemes. The UB allocations can simply be computed by ignoring the effect of ICI in all the cells i.e., by substituting $\xi_{n, l}=0$ in Algorithm 1:

$$
\mathrm{C}\left(\mathbf{A}_{l}, \mathbf{P}_{l}\right) \leq \frac{1}{L} \sum_{l=1}^{L} \sum_{k_{l}=1}^{K_{l}} \sum_{n=1}^{N} \alpha_{n, k_{l}, l} \log _{2}\left(1+\frac{p_{n, k_{l}, l} h_{n, k_{l}, l}}{\sigma^{2}}\right)
$$

Note that, allocations computed by Algorithm 1 are near optimal in the context of single cell scenarios [1]-[3]. The average network throughput revealed by the UB allocations could be highly optimistic for multi-cell systems.

\section{A Motivating Example}

Consider a motivating example with two cells, two users and two subcarriers. Each user can transmit with a maximum power of $1 \mathrm{~W}$. Assume $\mathbf{H}_{1}=\left[\begin{array}{llll}1 & 0.9 ; & 0.8 & 0.7\end{array}\right]$ and $\mathbf{H}_{2}=\left[\begin{array}{l}1 \\ 0\end{array}\right.$ $0.9 ; 0.80 .7]$. Single cell allocation strategies that aim to maximize the local throughput of each cell suggest $\mathbf{A}_{1}, \mathbf{P}_{1}$ and $\mathbf{A}_{2}, \mathbf{P}_{2}=\left[\begin{array}{llll}1 & 0 ; & 0 & 1\end{array}\right]$. Computing the UB using (7) results in $1.7655 \mathrm{bps} / \mathrm{Hz} / \mathrm{cell}$. Now, assuming the knowledge 
of interfering link gains at each BS, i.e., $\mathbf{G}_{12}=\left[\begin{array}{ll}0.9 & 0.2 ; 0.2\end{array}\right.$ $0.9]$ and $\mathbf{G}_{21}=\left[\begin{array}{lll}0.7 & 0.1 ; 0.1 & 0.7\end{array}\right]$. Computing the throughput again while keeping the single cell allocations and taking into account the interfering gains leads to an average network throughput of $1.1137 \mathrm{bps} / \mathrm{Hz}$. However, better allocations are possible if we consider $\mathbf{A}_{1}, \mathbf{P}_{1}$ and $\mathbf{A}_{2}, \mathbf{P}_{1}=\left[\begin{array}{llll}0 & 1 ; & 1 & 0\end{array}\right]$ as per the criterion discussed in Section IV which enhances the resulting average network throughput to $1.5977 \mathrm{bps} / \mathrm{Hz}$. The throughput calculated above can be increased further by computing the optimal powers (in high SINR regime) based on GP. Complexity Analysis: The $\left(n, k_{l}\right)$ pair at which the

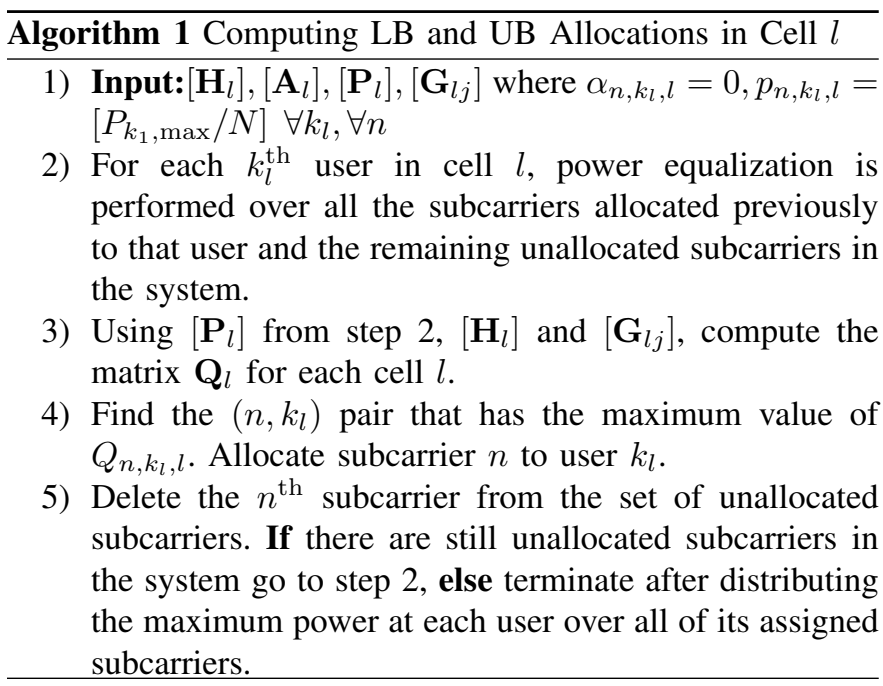

$Q_{n, k_{l}, l}$ becomes maximum is allocated (Step 4), which has a complexity of a two dimensional search, i.e., $O(K N)$. However, as soon as a subcarrier is assigned, each user updates its power as defined in Algorithm 1. This process iterates until all the subcarriers in all the cells are allocated and, thus, the time complexity of Algorithm 1 is $O\left(K N^{2}\right)$.

\section{Centralized Resource Allocation Schemes}

Assuming perfect knowledge of channel gains at a centralized controller, the optimal solution for (1) can be computed in the high SINR regime by an exhaustive search over all possible combinations of the allocations. For each possible allocation, optimum powers can be computed by transforming (1) into a geometric program (GP). Note that the power allocation problem is in itself a known non-convex problem for the general SINR regime. However, in high SINR regime the problem becomes a convex GP problem. For a given set of allocation variables and considering a high SINR regime, the objective function in (1) can be rewritten as follows:

$$
\underset{\mathbf{P}_{l}}{\operatorname{maximize}} \sum_{l=1}^{L} \sum_{k_{l}=1}^{K_{l}} \sum_{n=1}^{N} \alpha_{n, k_{l}, l} \log _{2}\left(\frac{p_{n, k_{l}, l} h_{n, k_{l}, l}}{\sigma^{2}+I_{n, l}}\right)
$$

Maximizing the SINRs is equivalent to minimizing the interference to signal ratio (ISNR):

$$
\underset{\mathbf{P}_{l}}{\operatorname{minimize}} \sum_{l=1}^{L} \sum_{k_{l}=1}^{K_{l}} \sum_{n=1}^{N} \alpha_{n, k_{l}, l} \log _{2}\left(\frac{\sigma^{2}+I_{n, l}}{p_{n, k_{l}, l} h_{n, k_{l}, l}}\right)
$$

Equivalently, (1) can be reformulated for high SINR regime and given allocation variables as follows:

$$
\begin{array}{ll}
\underset{\mathbf{P}_{l}}{\operatorname{minimize}} & \log _{2} \prod_{l=1}^{L} \prod_{k_{l}=1}^{K_{l}} \prod_{n=1}^{N}\left(\frac{\sigma^{2}+I_{n, l}}{p_{n, k_{l}, l} h_{n, k_{l}, l}}\right)^{\alpha_{n, k_{l}, l}} \\
\text { subject to } & \sum_{n=1}^{N} \alpha_{n, k_{l}, l} p_{n, k_{l}, l} \leq \mathrm{P}_{k_{l}, \max }, \quad \forall k_{l}, \forall l
\end{array}
$$

Note that the numerator in (10) is a posynomial and the denominator is a monomial, hence (10) is a GP problem in standard form that can be solved optimally through efficient interior point methods [11] after performing the logarithmic transformation of variables [12]. However, even for small dimensions, it is not recommendable to compute the optimal solution, due to the huge computational complexity $O\left(K^{L N}\right)$ associated with the exhaustive search based subcarrier allocation phase. In addition, the GP based power allocation method discussed above has two restrictions: high-SINR assumption and centralized time-consuming computations. Considering the high intricacy of implementing the optimal solution, a twostage centralized scheme is discussed in the following subsection.

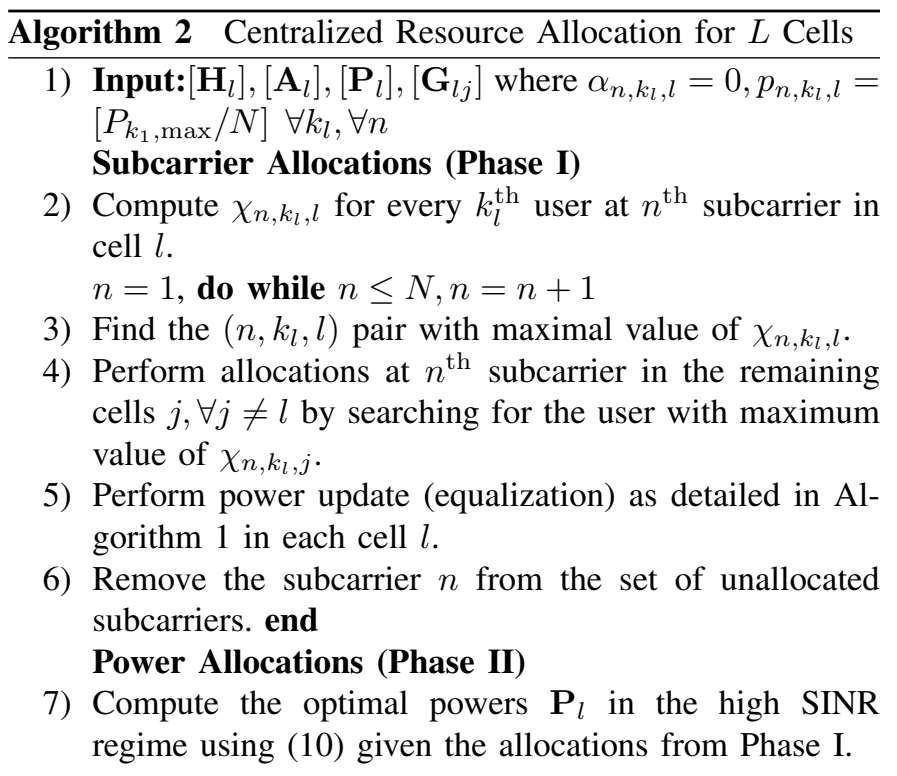

\section{A. Centralized Scheme A}

In the uplink, the subcarrier and the power allocation phases cannot be detached completely since the subcarrier allocation depends on the power with which a user may transmit at a specific subcarrier. In Phase I, we define the term $\chi_{n, k_{l}, l}=\frac{p_{n, k_{l}, l} h_{n, k_{l}, l}}{\sum_{j=1, j \neq l}^{L} P_{k_{l}, \max } g_{n, k_{l}, l j}}$ for the allocation of resources to users. This criterion guarantees the selection of the users who possess not only better power-gain product but also they offer less interference to the neighbor cells. The denominator $\sum_{j=1, j \neq l}^{L} P_{k_{l}, \max } g_{n, k_{l}, l j}$ accounts for the maximum interference that the $k_{l}^{\text {th }}$ user may cause to cell $j$. Even though, this criterion is heuristic, it improves the 
performance compared to the traditional C/I scheme (which gives nearly similar results as our lower bound). Once the subcarrier allocation is performed, the optimal powers can then be calculated for the high SINR regime as explained above or for the general SINR regime through solving a series of GPs using successive convex approximation which is a provably convergent heuristic [12].

Complexity Analysis: Initially we perform a three dimensional search over $\chi_{n, k_{l}, j}$ which has a complexity of $O(L K N)$. Next, a linear search is conducted in the cells other than the starting cell with a complexity of $O((L-1) K)$. The process iterates until all subcarriers are allocated, thus the total complexity of Phase I is $O\left(L K N^{2}+N K(L-1)\right)$. The complexity of Phase II is difficult to determine, however, it can be measured in terms of degree of difficulty (DoD) that in turn relies on the number of constraints and variables associated with the GP [13]. Since we are dealing with $L K$ power constraints and $L K N$ power variables, apparently it seems that implementing centralized GP/successive GP based schemes may not be a good choice for practical implementations. However, in order to reduce the complexity and DoD of GP we have developed the following less complex centralized scheme.

\section{B. Centralized Scheme B}

In this approach, instead of splitting the allocation procedure into two phases we combine them in the following manner. At first, the $n^{\text {th }}$ subcarrier is allocated in each cell $l$. Power allocation phase (based on equalization) is then executed as mentioned in Algorithm 2 (Steps 2-5). Note that before doing the next subcarrier allocation, we compute GP based power allocations for allocated users at subcarrier $n$. Setting the equalization based powers $p_{n, k_{l}, l_{\mathrm{eq}}}$ as the upper bound on $p_{n, k_{l}, l}$, we now define the less complex GP problem with the objective to maximize the throughput at the $n^{\text {th }}$ subcarrier.

$$
\begin{array}{ll}
\underset{p_{n, k_{l}, l}}{\operatorname{minimize}} & \log _{2} \prod_{l=1}^{L}\left(\frac{\sigma^{2}+I_{n, l}}{p_{n, k_{l}, l} h_{n, k_{l}, l}}\right) \\
\text { subject to } & p_{n, k_{l}, l} \leq \mathrm{p}_{n, k_{l}, l_{\mathrm{eq}}}, \quad \forall l
\end{array}
$$

Clearly, the resulting power for each competing user in different cells may not succeed in achieving the upper bound, due to the ICI effect. Thus, the remaining power of these users should be taken into account while doing the next round of allocations (i.e., immediately after computing the GP based powers, the remaining power is distributed equally among the remaining unallocated subcarriers of those users). The DoD of this scheme reduces to $L$ constraints and variables. Although this procedure restricts the degree of freedom offered by GP, numerical results show that the network throughput remains comparable with reduced complexity. The procedure is detailed in Algorithm 3.

\section{Semi-Distributed/Distributed Scheme}

In the semi-distributed strategy, we assume that every BS knows the interfering gains offered by its users to the neighboring BSs, i.e., $\sum_{j=1, j \neq l}^{L} P_{k_{l}, \max } g_{n, k_{l}, l j}$. Clearly, the interfering gains are based on path loss, shadowing and fading. Assuming the locations of local users at each BS, the path loss and shadowing gains of local users toward the first tier of interfering cells can be determined, however, the knowledge of fading gains is difficult to assume in practical scenarios. Thus, in the distributed approach, we compute our results without using the knowledge of fading interfering gains.

Each BS searches for the user with maximum value of $\chi_{n, k_{l}, l}$ in order to perform allocations. The allocation decisions are locally made at each BS (Phase I) and do not need collaboration. Once the allocations are decided, each cell shares them with all other interfering cells. The optimal powers can then be evaluated using dual decomposition methods by first performing the $\log$ transformation of the variables, i.e., $\ln p_{n, k_{l}, l}=\tilde{p}_{n, k_{l}, l}$ and $\ln p_{n, k_{j}, j}=\tilde{p}_{n, k_{j}, j}$, then adding auxiliary variable $\ln z_{n, j l}=\tilde{z}_{n, j l}$ where $z_{n, j l}=$ $\sum_{j=1, j \neq l}^{L} g_{n, k_{j}, j l} p_{n, k_{j}, j}$ in order to transfer the coupling in the objective to coupling into the constraints [12]. The problem defined in (9) can then be written as follows

$$
\begin{aligned}
\underset{\tilde{z}, \tilde{p}}{\operatorname{minimize}} & \sum_{l=1}^{L} \sum_{n=1}^{N} \log _{2}\left(\frac{\sigma^{2}+e^{\tilde{z}_{n, j l}}}{e^{\tilde{p}_{n, l}} h_{n, l}}\right) \\
\text { subject to } & \sum_{n=1}^{N} e^{\tilde{p}_{n, l}} \leq \mathrm{P}_{k_{l}, \max }, \quad \forall k_{l}, \forall l \\
e^{\tilde{z}_{n, j l}} & =\sum_{j=1, j \neq l}^{L} g_{n, j l} e^{\tilde{p}_{n, j}}, \forall n, \forall l
\end{aligned}
$$

However, (12) has high time complexity, thus, in this paper we present the dual decomposition of (11) which has a lesser time complexity.

$$
\begin{aligned}
& \underset{\tilde{z}, \tilde{p}}{\operatorname{minimize}} \sum_{l=1}^{L} \log _{2}\left(\frac{\sigma^{2}+e^{\tilde{z}_{n, j l}}}{e^{\tilde{e}_{n, l}} h_{n, l}}\right) \\
& \text { subject to } e^{\tilde{p}_{n, l}} \leq \mathrm{P}_{n, l, \mathrm{eq}}, \quad \forall l \\
& \qquad e^{\tilde{z}_{n, j l}}=\sum_{j=1, j \neq l}^{L} g_{n, j l} e^{\tilde{p}_{n, j}}
\end{aligned}
$$

Writing the Lagrange $\mathcal{L}\left(\tilde{p}_{n, l}, \tilde{z}_{n, j l}, \lambda_{k_{l}}, \eta_{n, l j}\right)$ for (13), and then splitting the problem into $L$ sub-problems yields (14) with local variables $\tilde{p}_{n, l}, \tilde{z}_{n, j l}, \lambda_{k_{l}}$ and coupling variables $\eta_{n, l j}$.

$$
\begin{array}{r}
\max \min \mathcal{L}_{l}\left(\tilde{p}_{n, l}, \tilde{z}_{n, j l}, \lambda_{k_{l}}, \eta_{n, l j}\right) \\
\text { subject to } \lambda_{k_{l}} \geq 0
\end{array}
$$

where $\lambda_{k_{l}}$ is the Lagrange multiplier for the inequality constraints and $\eta_{n, l j}$ are the consistency prices.

\section{Simulation Results and Performance EVALUATION}

A cellular OFDMA network is considered where the radius of each cell is assumed to be $R_{c}=0.5 \mathrm{~km}$. All users are placed at equally spaced angles from 0 to $2 \pi$. For demonstration purpose, the total number of users is assumed to be the same in all the cells. The maximum user transmit power is considered to be $1 \mathrm{~W}$. The channel gain is defined as 


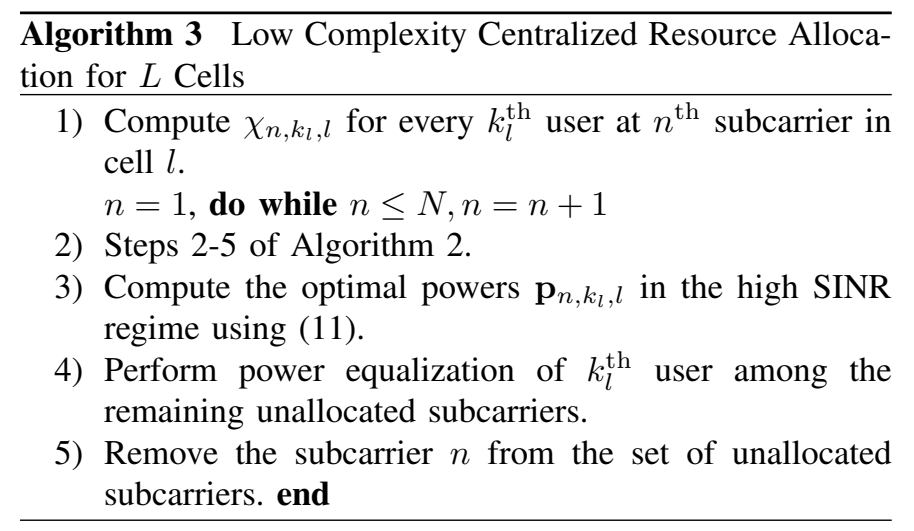

$h_{n, k_{l}, l}=\left(-122-10 \gamma \log _{10} d_{k_{l}, l}\right)-\mathcal{N}\left(0, \sigma^{2}\right)+10 \log _{10} F_{n, k_{l}, l}$. The first term denotes the path loss where $\gamma$ is the path loss exponent and is set equal to 3.8. The second term represents log-normal shadowing with a mean of $0 \mathrm{~dB}$ and a standard deviation of $8 \mathrm{~dB}$. The last factor, $F_{n, k_{l}, l}$ corresponds to Rayleigh fading. The bandwidth of the system is assumed to be $5 \mathrm{MHz}$ with a noise power spectral density of $2.07 \times 10^{-14} \mathrm{~W} / \mathrm{Hz}$ at each receiver. The channel conditions are assumed to be fixed during a frame. The interfering gains from $j^{\text {th }}$ interfering cell to the cell of interest $l$ are computed as $g_{n, k_{j}, l}=\left(-122-10 \gamma \log _{10} d_{k_{j}, l}\right)-\mathcal{N}\left(0, \sigma^{2}\right)+10 \log _{10} F_{n, k_{j}, l}$

In Table 1, we compare the performance and complexity of the centralized/distributed schemes for two cell scenario. The optimal solution is computed by an exhaustive search based subcarrier allocation phase detailed in Section IV. The results are taken after averaging over 100 channel realizations. The performance gap between the proposed centralized strategies and the optimal solution is negligible. However, this outcome may not remain valid for higher dimensions. In addition, the performance of the less complex centralized/distributed schemes remains comparable to the optimal solution. In order to get a more tighter LB, we compute the LB throughput by computing the optimal GP based power allocations given the LB subcarrier allocations from Algorithm 1.

In Fig.1 and Fig.2, we compare the performance of the centralized scheme B and the semi-distributed scheme for four cell and seven cells, respectively. The results have been taken after averaging over 10,000 channel realizations. We observe that for the 4-cell case, the centralized and semidistributed strategies give nearly similar results. However, as the number of cell increases the performance gain of the centralized scheme is evident. The LB throughput continues to decrease with the increase in number of interfering cells. Although, the UB is not tight, it provides the idea of a finite performance gap between the proposed schemes and optimal solution.

Another important observation in GP based optimal power allocation is that the sum $\sum_{n=1}^{N} p_{n, k_{l}, l}$ may or may not be equal to $P_{k_{l}, \text { max }}$ as dictated by the equalization based power allocation. Moreover, it may not (almost never) be optimal that two users transmit with powers suggested by power equalization based power allocations. Consider a channel realization $\mathbf{H}_{1}, \mathbf{H}_{2}=\left[\begin{array}{llll}0.3028 & 0.2503 ; 0.0388 & 0.1529\end{array}\right] \times 10^{-9}$

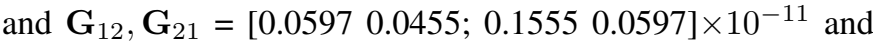
$[0.13400 .6860 ; 0.75380 .1935] \times 10^{-11}$, respectively. The equal power allocations dictates $\mathbf{P}_{1}=[00.5 ; 00.5]$ and $\mathbf{P}_{2}=$ $[0.50 ; 0.50]$ for semi-distributed scheme that leads to the average throughput of $11.8392 \mathrm{bps} / \mathrm{Hz}$. However, computing the optimal powers results in $\mathbf{P}_{1}=$ [0.00000.5326; 0.00000.4674] and $\mathbf{P}_{2}=\left[\begin{array}{llll}0.3840 & 0.0000 ; & 0.6160 & 0.0000\end{array}\right]$ which lead to a maximum average network throughput of $17.2734 \mathrm{bps} / \mathrm{Hz}$. This example demonstrates the significance of GP as well as centralized scheme B in which optimal powers are computed after each subcarrier allocation. Thus, the remaining unused power can be taken into account while allocating the next subcarrier. The significance of centralized scheme B is also shown in Fig. 3, where its performance is compared with the centralized scheme A (with and without power allocation stage). Clearly, without the prohibitively complex power allocation stage, the throughput of centralized scheme A may reduce significantly for high number of carriers, users and cells.

In Fig. 4 we have studied the performance of the distributed scheme, i.e., without considering the fading information for the 7-cell case. Results show that the performance is slightly degraded compared to the semi-distributed scheme (with fading knowledge).

\section{CONCLUSION}

In this paper, centralized and distributed resource allocation schemes are developed. Their performance is calibrated with the derived upper and lower bounds. Even though the upper bound is not tight, it exhibits a finite performance gap with the optimal solution. The centralized scheme A is more computationally complex. However, it provides a good calibration for the low complexity centralized/distributed schemes that are well-suited for practical scenarios.

\section{REFERENCES}

[1] K. Kim, Y. Han and S-L Kim, "Joint subcarrier and power allocation in uplink OFDMA systems," IEEE Communications Letters, vol. 9, no. 6 , pp. 526-528, June 2005.

[2] C. Ng and C. Sung, "Low complexity subcarrier and power allocation for utility maximization in uplink OFDMA systems," IEEE Transactions on Wireless Communications, vol. 7, no. 5 Part 1, pp. 1667-1675, 2008.

[3] Y. Ma, "Constrained rate-maximization scheduling for uplink OFDMA," Proceedings of IEEE Military Communications Conference, (MILCOM'07), pp. 1-7, Orlando, Florida, October 2007.

[4] E. Yaacoub, A. M. El-Hajj, and Z. Dawy, "Weighted ergodic sum-rate maximization in uplink OFDMA and its achievable rate region," IET Communications Journal, vol. accepted for publication.

[5] J. Huang, V. Subramanian, R. Agarwal, and R. Berry, "Joint scheduling and resource allocation in uplink OFDM Systems for broadband wireless access networks," IEEE Journal Selected Areas of Communications, vol. 27, pp. 226-234, February 2009.

[6] A. Abardo, A. Alessio, P. Detti and M. Moretti, "Centralized radio resource allocation for OFDMA cellular systems," Proceedings of IEEE International Conference on Communications, (ICC'07), pp. 5738 5743, Glasgow, Scotland, 24-28 June 2007.

[7] M. Moretti and A. Todini, "A resource allocator for the uplink of multicell OFDMA systems," IEEE Transactions on Wireless Communications, vol. 6, no. 8, pp. 2807-2812, August 2007.

[8] D. Gesbert and M. Kountouris, "Resource allocation in multicell wireless networks: Some capacity scaling laws," 5th International Symposium on Modeling and Optimization in Mobile, Ad Hoc and Wireless Networks and Workshops, 2007, pp. 1-7. 
TABLE I

AVERAGE NETWORK THROUGHPUT OF THE CENTRALIZED/SEMI-DISTRIBUTED SCHEMES FOR $L=2$ CELLS AND $N=6$ SUBCARRIERS/CELL

\begin{tabular}{|l||l|l|l|l|l|l|l||}
\hline \hline \multicolumn{1}{|l||}{} & $K=2$ & \multicolumn{1}{|l|}{$K=4$} & $K=6$ & \multicolumn{1}{l|}{ Complexity } \\
\cline { 2 - 7 } & $d_{i, k_{l}, l}=0.35 \mathrm{~km}$ & $d=0.45 \mathrm{~km}$ & $d=0.35 \mathrm{~km}$ & $d=0.45 \mathrm{~km}$ & $d_{i, k_{l}, l}=0.35 \mathrm{~km}$ & $d=0.45 \mathrm{~km}$ & \\
\hline \hline UB & 71.4294 & 62.5471 & 81.2473 & 72.6541 & 86.6465 & 79.045 & $O\left(K N^{2}\right)$ \\
Optimal & 46.7301 & 37.8545 & 54.8299 & 49.0265 & 61.1299 & 56.0121 & $O\left(K^{N L}\right)+D o D(L K N)$ \\
Centralized A & 46.1357 & 37.1154 & 54.4835 & 48.3071 & 60.4831 & 55.5927 & $O\left(L K N^{2}+(L-1) K N\right)+D o D(L K N)$ \\
Centralized B & 46.1434 & 37.0478 & 54.4945 & 48.1879 & 60.3795 & 55.4757 & $O\left(L K N^{2}+(L-1) K N\right)+D o D(L)$ \\
Semi-distributed & 45.7884 & 36.6796 & 53.6241 & 47.2564 & 59.1767 & 54.1465 & $O\left(K N^{2}\right)+D o D(L)$ \\
Distributed & 45.4544 & 36.294 & 52.8812 & 46.973 & 58.0834 & 53.5553 & $O\left(K N^{2}\right)+D o D(L)$ \\
LB & 41.3798 & 31.8322 & 46.7531 & 40.0884 & 50.4387 & 45.1596 & $O\left(K N^{2}\right)$ \\
\hline
\end{tabular}

[9] K. Yang, N. Prasad, X. Wang, "An auction approach to resource allocation in uplink multi-Cell OFDMA systems," Proceedings of Global Communications Conference, (GLOBECOM' 08), New Orleans, Louisinia, USA, November 30-December 4, 2008.

[10] E. Yaacoub and Z. Dawy, "A transparent pricing scheme for interference mitigation in uplink OFDMA with collaborative distributed scheduling," Proceedings of 17th International Conference on Telecommunications 2010 (ICT'10), Doha, Qatar, April 2010.

[11] M. Grant and S. Boyd, "CVX: MATLAB software for disciplined convex programming," 2007.

[12] M. Chiang, C. W. Tan, D. Palomar, D. O'Neill, and D. Julian, "Power control by geometric programming," IEEE Transactions on Wireless Communications, vol. 6, no. 7, pp. 2640-2651, July 2007.

[13] K. O. Kortanek, X. Xu, and Y.Ye, "An infeasible interior-point algorithm for solving primal and dual geometric programs," Mathematical Programming, vol. 76, pp. 155-181, 1997.

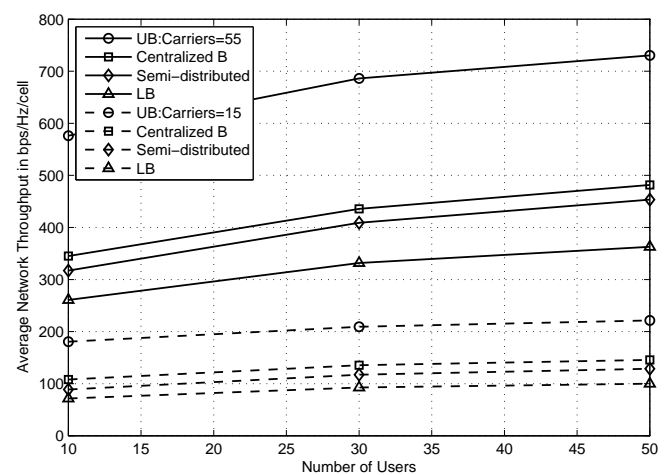

Fig. 1. Comparison of the centralized B and semi-distributed schemes for $L=4$ cell, $d=0.45 \mathrm{~km}$

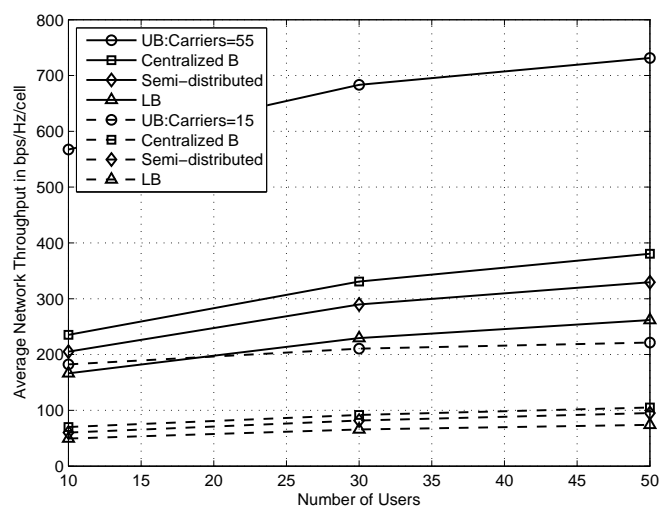

Fig. 2. Comparison of the centralized B and semi-distributed schemes for $L=7$ cell, $d=0.45 \mathrm{~km}$

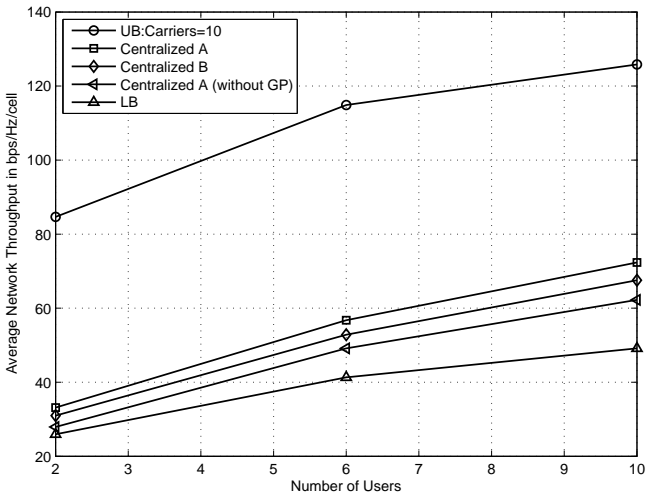

Fig. 4. Significance of GP based optimal power allocations in high SINR regime for $L=4$ cells, $N=10$ subcarriers $/$ cell and $d=0.45 \mathrm{~km}$

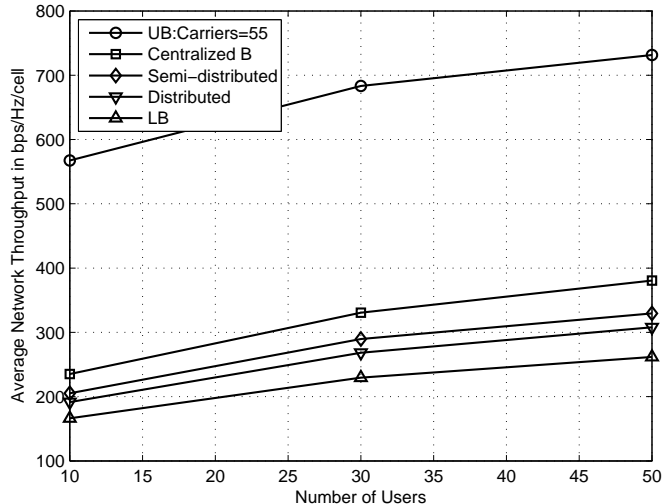

Fig. 3. Comparison of he distributed scheme (without fading knowledge) and the semi-distributed scheme (with fading knowledge) for $\mathrm{L}=7$ cells, $N=55$ subcarriers/cell and $d=0.45 \mathrm{~km}$ 